\title{
Fiber-delay-line-referenced optical frequency combs: three stabilization schemes
}

\author{
Kai Wang (王 凯) ${ }^{1}$, Haochen Tian (田昊晨) $)^{1^{*}}$ ， Fei Meng (孟飞) ${ }^{2,3}$ ，Baike Lin (林百科) ${ }^{2}$, Shiying Cao (曹士英) ${ }^{2}$ ， \\ Yihan Pi (皮一涵) ${ }^{1}$, Yan Han (韩 燕) ${ }^{1,4}$, Zhanjun Fang (方占军) $)^{2}$, Youjian Song (宋有建 $)^{1}$, and Minglie Hu (胡明列) ${ }^{1}$ \\ ${ }^{1}$ Ultrafast Laser Laboratory, Key Laboratory of Opto-Electronic Information Technical Science of Ministry of Education, School of Precision Instrument \\ and Opto-Electronics Engineering, Tianjin University, Tianjin 300072, China \\ ${ }^{2}$ Division of Time and Frequency Metrology, National Institute of Metrology, Beijing 100029, China \\ 3 Key Laboratory of Advanced Optical Communication System and Networks, School of Electronics Engineering and Computer Science, Peking University, \\ Beijing 100871, China \\ ${ }^{4}$ College of Physics and Optoelectronics, Taiyuan University of Technology, Taiyuan 030024, China
}

*Corresponding author: haochentian@tju.edu.cn

Received November 3, 2021 | Accepted November 18, 2021 | Posted Online December 14, 2021

We demonstrate the stabilization of an optical frequency comb (OFC) using a segment of fiber delay line as a reference. A mode-locked Er-doped fiber laser is phase locked to a kilometer-long fiber delay line using three different schemes. The short-term stability of the comb modes in the OFC stabilized by these schemes is obviously enhanced, down to the $10^{-12}$ level at millisecond average time. Among these three schemes, phase locking two bunches of comb modes in the OFC to the same fiber delay line exhibits the lowest residual phase noise. Fiber-delay-line-referenced OFCs can provide reliable laser sources in precise metrology owing to the advances of low cost, compactness, and high integration.

Keywords: optical frequency comb; delay line; frequency noise.

DOI: 10.3788/COL202220.021204

\section{Introduction}

Optical frequency combs (OFCs) emit optical pulse trains with millions of well-defined, mutually coherent, perfectly spaced comb modes ${ }^{[1-7]}$. This unique feature enables coherent phase linking among the optical, microwave, and terahertz regimes ${ }^{[8-10]}$. More importantly, the development of low-noise OFCs over the past two decades has experienced fascinating evolution, which facilitates advances of various applications such as ultra-low-noise microwave generation ${ }^{[11]}$, distance ranging $^{[12-14]}$, and dual-comb spectroscopy ${ }^{[15,16]}$.

Typically, noise at repetition rate $\left(f_{\text {rep }}\right)$ and carrier-envelope offset frequency $\left(f_{\text {ceo }}\right)$ in the laser is required to be reduced in order to obtain low-noise OFC operation. For $f_{\text {rep }}$ stabilization, a routine approach is to phase $\operatorname{lock} f_{\text {rep }}$, or it is harmonic to a radio reference, such as a cesium microwave atomic clock. Cavity length feedback enables $f_{\text {rep }}$ with stability of the $10^{-12}-10^{-13}$ level in $1 \mathrm{~s}$, determined by the noise performance of the referenced microwave oscillator ${ }^{[17]}$. An advanced approach is to trace one of the comb modes in the OFC to a narrow-linewidth optical reference to enhance the stability of $f_{\text {rep }}$. In this case, the fractional stability of $f_{\text {rep }}$ could be suppressed down to the $10^{-16}$ level in $1 \mathrm{~s}^{[18,19]}$.
For $f_{\text {ceo }}$ stabilization, in most cases, an $f-2 f$ interferometer is commonly used for $f_{\text {ceo }}$ detection. In this case, the $f_{\text {ceo }}$ signal is also referenced to a radio reference and able to inherit its stability ${ }^{[20,21]}$. Besides this, the phase noise of $f_{\text {ceo }}$ could be reduced passively or by phase locking to the laser's $f_{\text {rep }}$ as well ${ }^{[22,23]}$. Another significant approach that needs to be mentioned here is to phase lock two comb modes in the OFC to two optical references separately ${ }^{[24]}$. In this way, all the comb modes are capable of inheriting stability of the low-noise optical references, thus, resulting in a stabilized $f_{\text {ceo }}$.

Besides radio frequency references and optical references, kilometer-long fiber delay lines are also proved to be reliable references for short-term stability enhancement in OFCs recently ${ }^{[25-27]}$. In this Letter, we improve the short-term stability of the comb modes in OFCs using a fiber delay line as a reference. Two schemes are proposed, and their stabilization performances are compared with our prior work ${ }^{[28]}$. We labeled three stabilization schemes as follows: (i) $1566 \mathrm{~nm}$ comb modes are stabilized to a fiber delay line, and $f_{\text {rep }}$ is stabilized to a rubidium microwave atomic clock; (ii) $1566 \mathrm{~nm}$ comb modes and $f_{\text {rep }}$ are stabilized to a fiber delay line; (iii) $1526 \mathrm{~nm}$ and $1566 \mathrm{~nm}$ comb modes are stabilized to a fiber delay line. The short-term stability of the OFCs stabilized by these schemes is obviously enhanced, 
down to the $10^{-12}$ level at millisecond average time. The third scheme shows the lowest residual phase noise by seizing two bunches of comb modes in the OFC to the fiber delay line at the same time. Fiber-delay-line-referenced OFCs provide reliable laser sources in low-noise-OFC-based precise metrology and other applications.

The target OFC modes' frequency noise detection and stabilization are based on the delayed self-heterodyne (DSH) method, which is a state-of-the-art technique in CW lasers' frequency noise detection and suppression. Procedures for comb modes' frequency noise reduction using DSH are as follows. A bunch of comb modes (centered at $\lambda_{1}$ or $\lambda_{2}$ ) of the OFC under stabilization are filtered out by a dense wavelength division multiplexer (DWDM) and sent into a home-built asymmetric fiber interferometer (AFI), as shown in Fig. 1. Detailed configuration of the AFI could be found in our previous publication ${ }^{[28,29]}$. The heterodyne beat from the interferometer carries the frequency noise $p \cdot \delta\left[\tau\left(n f_{\text {rep }}+f_{\text {ceo }}+2 f_{\text {mod }}\right)\right]$, where $p$ accounts for the entire number of comb lines within the DWDM's bandwidth. $n$ represents the mode number. $\tau$ is the round-trip delay time in the interferometer. $\delta\left[\tau\left(n f_{\text {rep }}+f_{\text {ceo }}+2 f_{\text {mod }}\right)\right]$ represents the frequency noise of the comb lines. $f_{\text {mod }}$ represents the acoustic optical frequency shifter's modulation frequency in the interferometer. The kilometer-long optical fiber delay line in the interferometer magnifies the frequency noise of the comb modes by a factor of $\tau$. Error signal containing comb-line frequency noise $p$. $\delta\left[\tau\left(n f_{\text {rep }}+f_{\text {ceo }}\right)\right]$ could be generated by mixing the heterodyne beat with $2 f_{\text {mod }}$. Through feeding back this error signal on either intra-cavity or extra-cavity actuators using a proportional-integral-derivative (PID) controller, the frequency noise of the filtered comb lines could be effectively suppressed.

Repetition rate stabilization of the target OFC requires the participation of two separated bunches of comb modes, e.g., $m$ th and $n$th comb modes. Two beat notes carrying comb-line frequency noise $p \cdot \delta\left[\tau\left(m f_{\text {rep }}+f_{\text {ceo }}+2 f_{\text {mod }}\right)\right]$ and $p$. $\delta\left[\tau\left(n f_{\text {rep }}+f_{\text {ceo }}+2 f_{\text {mod }}\right)\right]$ are detected by two low-noise photodiodes separately. After $f_{\text {ceo }}$ and $f_{\text {mod }}$ are rejected by a frequency mixer, the repetition rate noise, $\delta(m-n) f_{\text {rep }}$, could be obtained, as shown in Fig. 1. Similarly, feeding back this error signal to cavity length modulation or pump current modulation using a commercial PID servo could suppress the repetition noise of the OFC. More details in $f_{\text {rep }}$ noise stabilization have been

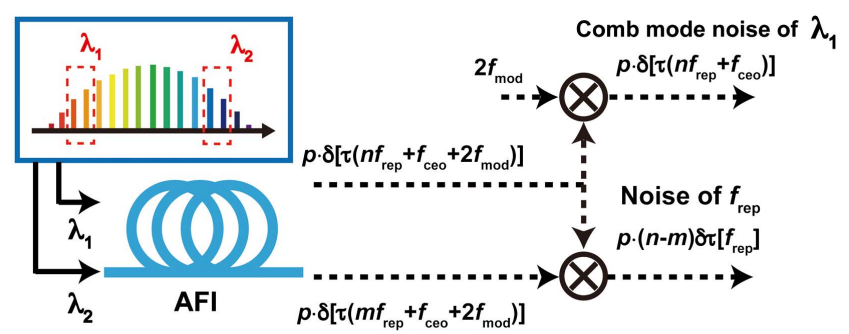

Fig. 1. Principle of comb mode noise detection and repetition rate noise detection based on DSH method. AFI, asymmetric fiber interferometer. reported in literature ${ }^{[30]}$. In practice, the length of fiber delay in the fiber interferometer needs to be carefully chosen. Longer fiber delay leads to higher discrimination sensitivity in the DSH setup for frequency noise detection. However, the longer the fiber delay line is, the lower frequencies of the null frequency points in the transfer function are. For example, when the fiber delay line is $10-\mathrm{km}$-long, null frequencies would gradually arise at the $10 \mathrm{kHz}$ Fourier frequency range, which would restrain the phase-locking bandwidth. Consequently, in both comb mode and repetition rate noise reduction, kilometer-long fiber delay line is typically chosen under the consideration of this tradeoff. Three distinct stabilization schemes will be introduced in detail in what follows.

\section{Comb Modes at $1566 \mathrm{~nm}$ Are Stabilized to a Fiber Delay Line, and $f_{\text {rep }}$ Is Stabilized to a Signal Generator}

Our first stabilization scheme is to phase lock the repetition rate of the OFC to a signal generator, which is referenced by a rubidium microwave atomic clock. Meanwhile, $1566 \mathrm{~nm}$ comb modes are phase locked to the fiber delay line. The mode-locked laser under stabilization is a home-built Er-fiber mode-locked laser with $205 \mathrm{MHz}$ repetition rate. As shown in Fig. 2(a), the optical pulses from the laser are detected by a high-speed photodiode (Electro-Optics Technology, ET-3000A). As a result, the repetition rate's fifth harmonic is detected and filtered out by a band pass filter (center frequency of $1.025 \mathrm{GHz}$ ). Through pump power feedback using a PID controller, the fifth repetition rate harmonic is phase locked to the signal generator. After phase locking, stability of the repetition rate inherits that of the microwave reference, which is at the $10^{-11}$ level in $1 \mathrm{~s}$ and the $10^{-13}$ level in $100 \mathrm{~s}$. The locking bandwidth of the repetition rate is $\sim 10 \mathrm{kHz}$. Then, comb modes around $1566 \mathrm{~nm}$ are filtered out and sent into the asymmetric Michelson fiber interferometer. The output heterodyne beat carrying the frequency noise of

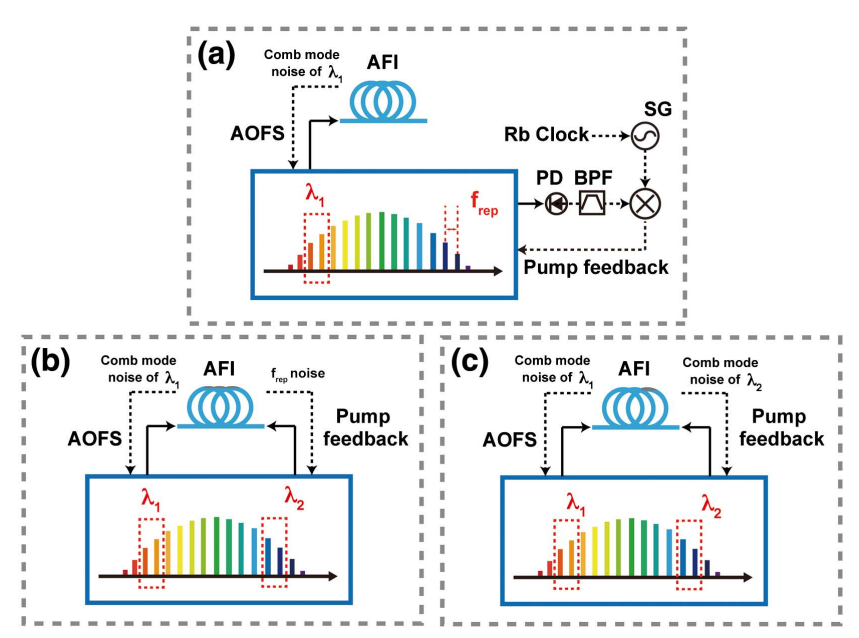

Fig. 2. Three experimental schemes of the fiber-delay-line-referenced OFCs. PD, photodetector; BPF, band-pass filter; SG, signal generator; AFI, asymmetric fiber interferometer. 
filtered comb modes is mixed with $2 f_{\text {mod }}$ and further fed back on an extra-cavity acousto-optic frequency shifter (AOFS) with $40 \mathrm{kHz}$ modulation bandwidth. The phase-locking bandwidth is mainly limited by the null frequency points in the fiber delay line. To this end, both degrees of freedom of the target OFC, $f_{\text {rep }}$ and $f_{\text {ceo }}$, are fully stabilized.

To evaluate the noise performance of the fully stabilized OFC, out-of-loop phase noise measurement is conducted. In this case, we beat a certain comb mode in the OFC with a commercial $1542 \mathrm{~nm}$ CW laser (Stable Laser Systems). The typical linewidth of the CW laser is $1 \mathrm{~Hz}$. Since the reference CW laser has negligible noise compared to stabilized OFC, the phase noise and Allan deviation of the resulting beat signal $\left(f_{\text {beat }}\right)$ represent the noise performance of the comb mode at $1542 \mathrm{~nm}$ in the OFC. A frequency analyzer is applied to record the frequency fluctuation of $f_{\text {beat }}$ with gate time of $100 \mu \mathrm{s}$. The fractional Allan deviation of $f_{\text {beat }}$ is calculated, as shown in Fig. 3(a). When the OFC is free-running, the Allan deviation of $f_{\text {beat }}$ is at the $10^{-11}$ level, as represented in the gray curve in Fig. 3(a). After phase locking, the short-term stability ( $100 \mu$ s to $10 \mathrm{~ms}$ range) of $f_{\text {beat }}$ is obviously enhanced by one order of magnitude, as shown in the orange curve in Fig. 3(a). Allan deviation averages down as $\tau^{-1 / 2}$ until $10 \mathrm{~ms}$ and reaches $4.4 \times 10^{-12}$ at $6.4 \mathrm{~ms}$. However, in the long-time scale, $f_{\text {beat }}$ starts to drift. The singleside phase noise power spectral density (PSD) of $f_{\text {beat }}$ is characterized by a signal source analyzer (Keysight, E5052b). Accordingly, from comparison of residual phase noise PSD of $f_{\text {beat }}$ between the free-running condition and phase-locked condition, the phase noise has been suppressed by one order of magnitude at $<10 \mathrm{kHz}$ Fourier frequency range. The suppression bandwidth is $\sim 40 \mathrm{kHz}$, where a servo bump at this frequency in the phase noise curve exists, as shown in the orange curve in Fig. 3(b).

\section{Comb Modes at $1566 \mathrm{~nm}$ and $f_{\text {rep }}$ Are Stabilized to a Fiber Delay Line}

The second stabilization scheme is to phase lock both $f_{\text {rep }}$ and $1566 \mathrm{~nm}$ comb modes in the OFC to the fiber delay line, as illustrated in Fig. 2(b). Repetition rate stabilization to the fiber delay line needs two heterodyne beats at $1526 \mathrm{~nm}$ and $1566 \mathrm{~nm}$. Here, the $1566 \mathrm{~nm}$ heterodyne beat is split by a 50:50 coupler. Halfpower of the beat is utilized for comb modes stabilization, which is the same as scheme (i). The rest power of the beat is directed to the local oscillator ( $\mathrm{LO}$ ) port of a mixer. It should be noted that the $\mathrm{LO}$ port of the mixer we used here requires at least $7 \mathrm{dBm}$ power input to fully drive the mixer with low conversion loss. The $1566 \mathrm{~nm}$ beat needs to be amplified by an electronic amplifier before going into the mixer's LO port. At the same time, the $1526 \mathrm{~nm}$ beat goes into the RF port of the mixer. The result from the intermediate frequency (IF) port characterizes the $f_{\text {rep }}$ noise of the target OFC. Regarding this as the error signal, the repetition rate could be stabilized to the fiber delay line via pump current feedback using a PID controller. (a)

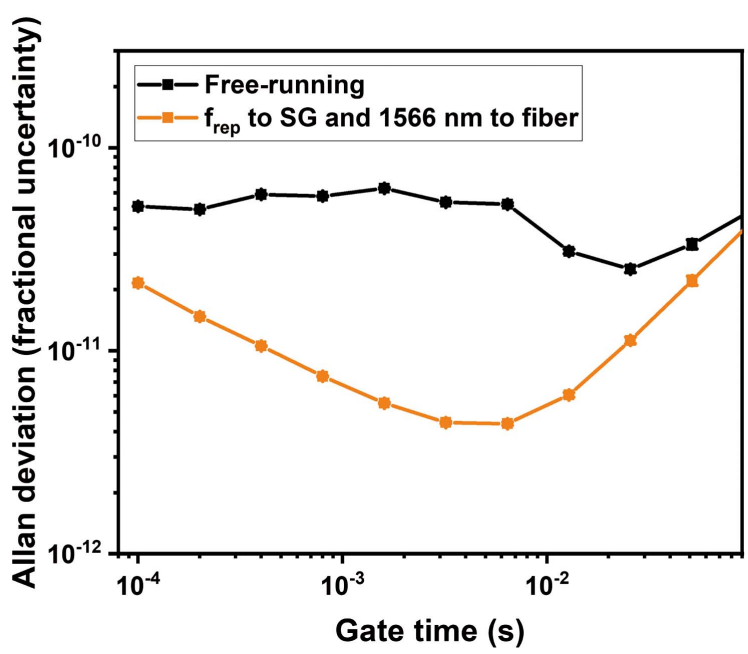

(b)

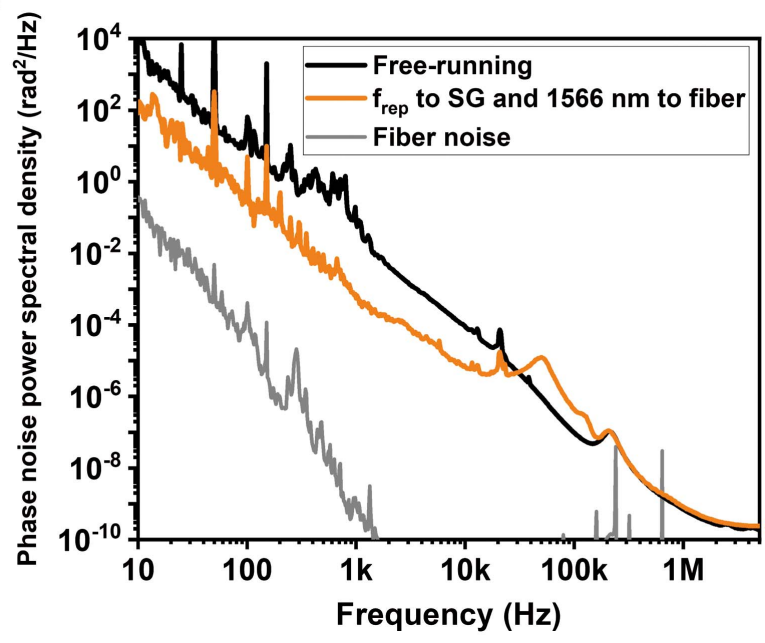

Fig. 3. (a) Frequency stability and (b) out-of-loop phase noise PSD of $1542 \mathrm{~nm}$ comb mode using scheme (i).

After the close of two phase-locked loops, the out-of-loop measurement of $f_{\text {beat }}$ is conducted, as a similar manner in scheme (i). The corresponding Allan deviation and phase noise PSD are shown in Figs. 4(a) and 4(b), respectively. The results are very similar to scheme (i), showing enhanced short-term stability.

\section{Comb Modes at $1526 \mathrm{~nm}$ and $1566 \mathrm{~nm}$ Are Stabilized to a Fiber Delay Line}

The third stabilization scheme is inspired by the phase locking scheme from Ref. [24], where both $1526 \mathrm{~nm}$ and $1566 \mathrm{~nm}$ comb modes are stabilized to the fiber delay line, as illustrated in Fig. 2(c). The error signal from the $1566 \mathrm{~nm}$ heterodyne beat is fed back upon the extra-cavity AOFS through a PID servo for $f_{\text {ceo }}$ stabilization. On the other hand, the error signal from the $1526 \mathrm{~nm}$ heterodyne beat is fed back upon pump current modulation through a proportional-integral (PI) servo for repetition rate stabilization. The out-of-loop measurement results 
(a)

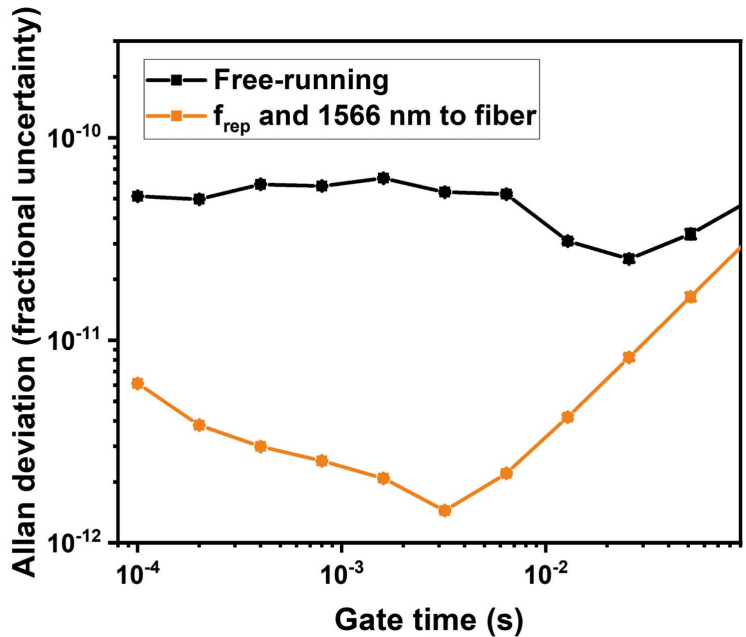

(b)

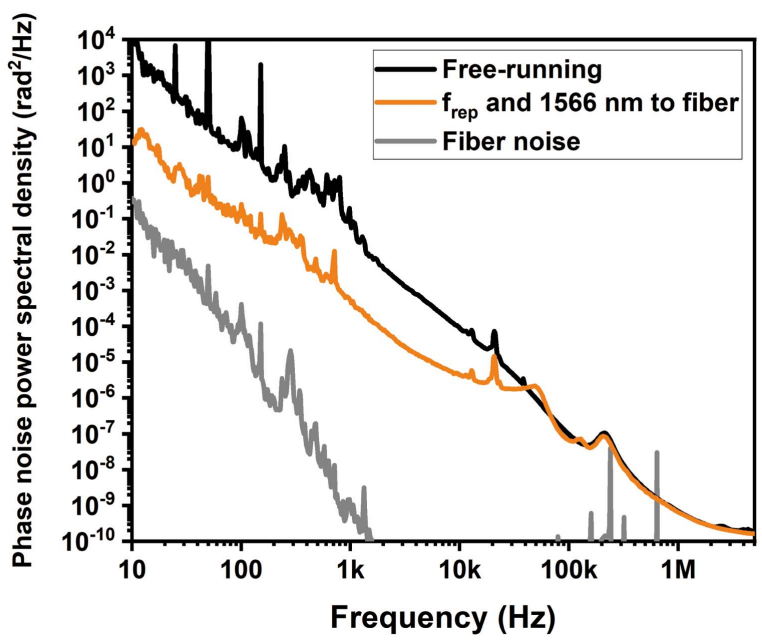

Fig. 4. (a) Frequency stability and (b) out-of-loop phase noise PSD of $1542 \mathrm{~nm}$ comb mode using scheme (ii).

are presented in Fig. 5. After phase locking, Allan deviation of $f_{\text {beat }}$ reaches $2.7 \times 10^{-12}$ at $6.4 \mathrm{~ms}$. Phase noise PSD is presented in Fig. 5(b). After phase locking, the integrated residual phase noise is $666 \mathrm{mrad}$ (from $10 \mathrm{MHz}$ to $1 \mathrm{kHz}$ ) and $1.5 \mathrm{rad}$ (from $5 \mathrm{MHz}$ to $100 \mathrm{~Hz}$ ). The phase noise of the fiber delay line could also be characterized using the commercial CW laser. The CW laser is directed into the AFI. The output heterodyne beat is detected, filtered, amplified, and finally mixed with $2 f_{\text {mod }}$. Through division of the mixing result's voltage PSD by the fiber delay line's transfer function ${ }^{[28,29]}$, phase noise of the fiber delay line could be retrieved, as represented by the gray curves in Figs. 3(b), 4(b), and 5(b).

To summarize, we compared three distinct stabilization schemes using a fiber delay line as a reference: (i) $1566 \mathrm{~nm}$ comb modes are stabilized to a fiber delay line and the repetition rate is stabilized to a signal generator; (ii) $1566 \mathrm{~nm}$ comb modes and repetition rate are stabilized to a fiber delay line; (iii) $1526 \mathrm{~nm}$ and $1566 \mathrm{~nm}$ comb modes are stabilized to a fiber delay line. The short-term stability of the OFCs stabilized by these schemes is obviously enhanced, down to the $10^{-12}$ level at millisecond (a)

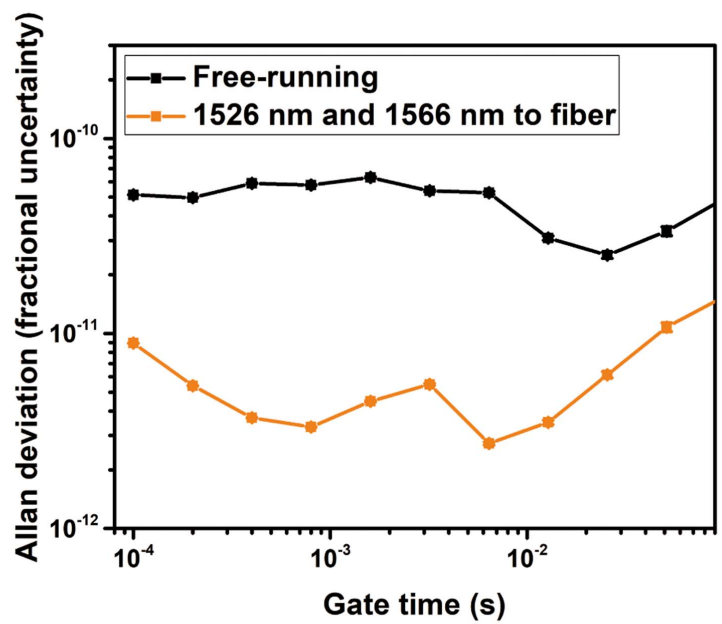

(b)

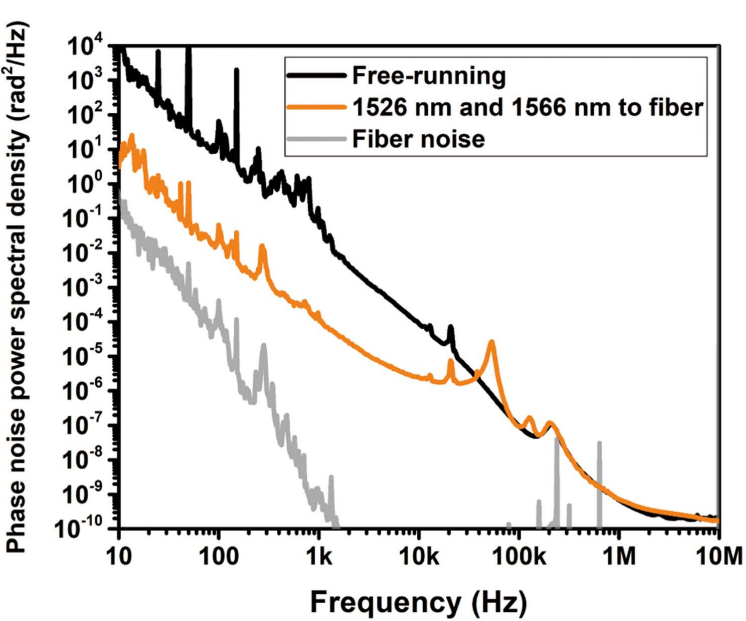

Fig. 5. (a) Frequency stability and (b) out-of-loop phase noise PSD of $1542 \mathrm{~nm}$ comb mode using scheme (iii).

average time. The noise performance comparison between these three cases is shown in Table 1. In these three cases, the lowest Allan deviations are nearly at the same level. The short-term stability is better than that of most microwave atomic clocks. The phase noise spectra show some distinction. In schemes (i) and (ii), fifth $f_{\text {rep }}\left(\sim 1 \mathrm{GHz}\right.$, radio frequency domain) and $(m-n) f_{\text {rep }}$ ( $\sim 5 \mathrm{THz}$, terahertz frequency domain) are phase locked, respectively. The stability would be degraded by certain factors when transferred to $1540 \mathrm{~nm}$ comb modes $(194.8 \mathrm{THz}$, optical

Table 1. Noise Performance Comparison of Three Stabilization Schemes.

\begin{tabular}{lcc}
\hline $\begin{array}{c}\text { Stabilization } \\
\text { Scheme }\end{array}$ & $\begin{array}{c}\text { Lowest Allan } \\
\text { Deviation }\end{array}$ & $\begin{array}{c}\text { Integrated Phase Noise } \\
\text { (from } 5 \mathrm{MHz} \text { to } 100 \mathrm{~Hz} \text { ) (rad) }\end{array}$ \\
\hline (i) & $4.4 \times 10^{-12}$ at $6.4 \mathrm{~ms}$ & 7.7 \\
(ii) & $1.4 \times 10^{-12}$ at $3.2 \mathrm{~ms}$ & 3.7 \\
(iii) & $2.7 \times 10^{-12}$ at $6.4 \mathrm{~ms}$ & 1.5 \\
\hline
\end{tabular}


frequency domain). However in scheme (iii), two phase-locked loops directly seized two bunches of comb lines in the optical frequency domain $(196.6 \mathrm{THz}$ and $191.57 \mathrm{THz})$. Thus, the third scheme characterizes the lowest integrated phase noise. Therefore, we think it is the most practical stabilization scheme. It should be noted that the advantage of using the fiber delay line as a reference is its superior short-term stability. The long-term stability in all the three cases is gradually deteriorating, which is affected by the slow drifting of the fiber delay line due to the environmental variations. Additional temperature control may partly overcome this problem. Fiber-delay-line-referenced OFCs get rid of $f-2 f$ interferometers and low-noise optical references. The entire phase-locking system is with low-cost, compact, and highly integrated. More importantly, the stabilization could be realized with only hundred-milliwatt average power. Thus, this derived phase-locking method is not restrained for mode-locked lasers' stabilization, but could be also applied in noise suppression of electro-optic (EO) combs, quantum cascade laser combs, micro-resonator combs, etc. All of these merits enable the presented OFC to be a practical laser source in low-noise-OFC-based precise metrology, microwave generation, and dual-comb spectroscopic applications.

\section{Acknowledgement}

This work was supported by the National Natural Science Foundation of China (Nos. 61975144 and 61827821) and the Tianjin Research Program of Application Foundation and Advanced Technology of China (No. 17JCJQJC43500).

\section{References}

1. T. Fortier and E. Baumann, " 20 years of developments in optical frequency comb," Comms. Phys. 2, 153 (2019).

2. M. Giunta, M. Fischer, W. Hänsel, T. Steinmetz, M. Lessing, S. Holzberger, C. Cleff, T. W. Hänsch, M. Mei, and R. Holzwarth, "20 years and 20 decimal digits: a journey with optical frequency combs," IEEE Photon. Technol. Lett. 31, 1898 (2019).

3. J. Kim and Y. Song, "Ultralow-noise mode-locked fiber lasers and frequency combs: principles, status, and applications," Adv. Opt. Photonics 8, 465 (2016).

4. H. Tian, Y. Song, and M. Hu, "Noise measurement and reduction in modelocked lasers: fundamentals for low-noise optical frequency combs," Appl. Sci. 11, 7650 (2021).

5. W. Xia and X. Chen, "Recent developments in fiber-based optical frequency comb and its applications," Meas. Sci. Technol. 27, 041001 (2016).

6. Y. Chang, T. Jiang, Z. Zhang, and A. Wang, "All-fiber Yb: fiber frequency comb," Chin. Opt. Lett. 17, 053201 (2019).

7. G. Yang, H. Shi, Y. Yao, H. Yu, Y. Jiang, A. Bartels, and L. Ma, "Long-term frequency-stabilized optical frequency comb based on a turnkey Ti:sapphire mode-locked laser," Chin. Opt. Lett. 19, 121405 (2021).

8. S. A. Diddams, D. J. Jones, J. Ye, S. T. Cundiff, and J. L. Hall, "Direct link between microwave and optical frequencies with a $300 \mathrm{THz}$ femtosecond laser comb," Phys. Rev. Lett. 84, 5102 (2000).

9. M. Giunta, W. Hänsel, M. Fischer, M. Lezius, T. Udem, and R. Holzwarth, "Real-time phase tracking for wide-band optical frequency measurements at the 20th decimal place," Nat. Photonics 14, 44 (2019).
10. L. Consolino, A. Taschin, P. Bartolini, S. Bartalini, P. Cancio, A. Tredicucci, H. E. Beere, D. A. Ritchie, R. Torre, M. S. Vitiello, and P. De Natale, "Phaselocking to a free-space terahertz comb for metrological-grade terahertz lasers," Nat. Commun. 3, 1040 (2012).

11. T. M. Fortier, A. Rolland, F. Quinlan, F. N. Baynes, A. J. Metcalf, A. Hati, A. D. Ludlow, N. Hinkley, M. Shimizu, T. Ishibashi, J. C. Campbell, and S. A. Diddams, "Optically referenced broadband electronic synthesizer with 15 digits of resolution," Laser Photonics Rev. 10, 780 (2016).

12. H. Shi, Y. Song, R. Li, Y. Li, H. Cao, H. Tian, B. Liu, L. Chai, and M. Hu, "Review of low timing jitter mode-locked fiber lasers and applications in dual-comb absolute distance measurement," Nanotechnol. Precis. Eng. 1, 205 (2018).

13. W. Kim, J. Jang, S. Han, S. Kim, J. S. Oh, B. S. Kim, Y.-J. Kim, and S.-W. Kim, "Absolute laser ranging by time-of-flight measurement of ultrashort light pulses [Invited]," J. Opt. Soc. Am. A 37, B27 (2020).

14. Y. Li, Y. Cai, R. Li, H. Shi, H. Tian, M. He, Y. Song, and M. Hu, "Large-scale absolute distance measurement with dual free-running all-polarizationmaintaining femtosecond fiber lasers," Chin. Opt. Lett. 17, 091202 (2019).

15. I. Coddington, N. Newbury, and W. C. Swann, "Dual-comb spectroscopy," Optica 3, 414 (2016).

16. R. Liao, H. Tian, W. Liu, R. Li, Y. Song, and M. Hu, "Dual-comb generation from a single laser source: principles and spectroscopic applications towards mid-IR: a review," J. Phys. Photonics 2, 042006 (2020).

17. T. R. Schibli, K. Minoshima, F.-L. Hong, H. Inaba, A. Onae, H. Matsumoto, I. Hartl, and M. E. Fermann, "Frequency metrology with a turnkey all-fiber system," Opt. Lett. 29, 2467 (2004).

18. J. Millo, R. Boudot, M. Lours, P. Y. Bourgeois, A. N. Luiten, Y. Le Coq, Y. Kersalé, and G. Santarelli, "Ultra-low-noise microwave extraction from fiber-based optical frequency comb," Opt. Lett. 34, 3707 (2009).

19. T. M. Fortier, M. S. Kirchner, F. Quinlan, J. Taylor, J. C. Bergquist, T. Rosenband, N. Lemke, A. Ludlow, Y. Jiang, C. W. Oates, and S. A. Diddams, "Generation of ultrastable microwaves via optical frequency division," Nat. Photonics 5, 425 (2011).

20. L. C. Sinclair, J.-D. Deschênes, L. Sonderhouse, W. C. Swann, I. H. Khader, E. Baumann, N. R. Newbury, and I. Coddington, "Invited article: a compact optically coherent fiber frequency comb," Rev. Sci. Instrum. 86, 081301 (2015).

21. M. Endo, T. D. Shoji, and T. R. Schibli, "Ultralow noise optical frequency combs," IEEE J. Sel. Top. Quantum Electron. 24, 1102413 (2018).

22. G. Krauss, D. Fehrenbacher, D. Brida, C. Riek, A. Sell, R. Huber, and A. Leitenstorfer, "All-passive phase locking of a compact Er:fiber laser system," Opt. Lett. 36, 540 (2011).

23. S. Okubo, A. Onae, K. Nakamura, T. Udem, and H. Inaba, "Offset-free optical frequency comb self-referencing with an $\mathrm{f}-2 \mathrm{f}$ interferometer," Optica $\mathbf{5}$, 188 (2018).

24. I. Coddington, W. C. Swann, and N. R. Newbury, "Coherent multiheterodyne spectroscopy using stabilized optical frequency combs," Phys. Rev. Lett. 100, 013902 (2008).

25. D. Kwon, C. Jeon, J. Shin, M. Heo, S. E. Park, Y. Song, and J. Kim, "Referencefree, high-resolution measurement method of timing jitter spectra of optical frequency combs,” Sci. Rep. 7, 40917 (2017).

26. D. Kwon, I. Jeon, W. Lee, M. Heo, and J. Kim, "Generation of multiple ultrastable optical frequency combs from an all-fiber photonic platform," Sci. Adv. 6, eaax4457 (2020).

27. M. Wada, F. Hong, and H. Inaba, "Frequency noise measurement and its uncertainty estimation of an optical frequency comb using a delay line interferometer," Meas. Sci. Technol. 31, 125012 (2020).

28. H. Tian, F. Meng, K. Wang, B. Lin, S. Cao, Z. Fang, Y. Song, and M. Hu, "Optical frequency comb stabilized to a fiber delay line," Appl. Phys. Lett. 119, 121106 (2021).

29. H. Tian, W. Yang, D. Kwon, R. Li, Y. Zhao, J. Kim, Y. Song, and M. Hu, "Optical frequency comb noise spectra analysis using an asymmetric fiber delay line interferometer," Opt. Express 28, 9232 (2020).

30. D. Kwon and J. Kim, "All-fiber interferometer-based repetition-rate stabilization of mode-locked lasers to $10^{-14}$-level frequency instability and 1-fslevel jitter over 1s," Opt. Lett. 42, 5186 (2017). 